\title{
Estimation of anisotropy parameters in Orthorhombic media
}

\author{
Pavan Elapavuluri and John Bancroft
}

\begin{abstract}
In order to extend seismic processing techniques to anisotropic media, it is essential that we have a measure of the different anisotropy parameters. The purpose of this study is to estimate Thomsen's parameters for P-wave, namely $\epsilon$ and $\delta$ for transversely isotropic (TI) media and also to extend to orthorhombic media by using the shifted hyperbola NMO equation. In order to estimate these parameters an estimate of vertical velocity is usually needed. We propose a method for estimating the anisotropy parameters independent of the vertical velocity; in fact we also estimate vertical velocity. This method was tested on the data collected over a synthetic model with both vertical transverse isotropy (VTI) and orthorhombic symmetries. This method fails when $\epsilon=\delta$, i.e if the symmetry of the medium is elliptical.
\end{abstract}

\section{INTRODUCTION}

In order to study and understand the complex Earth, exploration geophysicists made many assumptions. One of them is that earth is perfectly isotropic while it is fundamentally anisotropic (Thomsen, 1986). Backus (1962) stated that if the layered earth is probed with an elastic wave of wavelength much longer than the typical layer thickness the wave propagates through this medium as it were in a homogeneous and anisotropic medium. Alkhalifah and Tsvankin (1994) used an inversion scheme over the non-hyperbolic equation to estimate Thomsen's anisotropic parameters and they found out the inversion procedure to estimate anisotropy parameters along with vertical velocity is highly unconstrained.

In this paper we will be detailing the procedure to estimate the anisotropy parameters which characterize orthorhombic media. Determination of $\delta$ (the short offset effect) is relatively easy but $\epsilon$ (long offset effect) is difficult and needs a measure of horizontal velocity, which is difficult to measure. In this study the long offset moveout information is used for $\epsilon$ estimation. Usually Dix type normal moveout (NMO) (Castle, 1994) correction at long offsets is not very accurate and it even worsens when there is anisotropy present. The Shifted hyperbola NMO (SHNMO) equation is more accurate at longer offsets than Dix NMO equation. Therefore by using the Shifted hyperbola NMO equation to correct long offset data we get a better estimation of RMS velocity (therefore better interval velocity) and so a better estimation of $\delta$ and $\epsilon$.

\section{ANISOTROPY AND ITS MEASUREMENT}

The most common measure of $\mathrm{P}$ wave anisotropy, is the ratio between the horizontal and vertical $\mathrm{P}$ wave velocities, typically between 1.05 and 1.1 and is often as large as 1.2 (Sheriff, 1991). Thomsen (1986) introduced a more effective and scientific measure of anisotropy. He introduced these constants as effective parameters of measure of anisotropy. According to Thomsen, $\delta$ is the most critical measure of anisotropy and it does not involve the horizontal velocity at all in its definition. Therefore measuring $\delta$ is very important for processes like depth imaging. Measurement of $\delta$ requires the measurement of vertical 
velocity. In this paper we show that vertical velocity can be estimated from surface seismic data itself; in the same way horizontal velocity can also be estimated from surface seismic data and thus $\epsilon$.

The main technique used to estimate the seismic velocity is to fit a moveout (NMO) hyperbola to the traveltime curve. The main methods used to perform the velocity analysis are Normal Moveout Analysis and Shifted hyperbola NMO (SHNMO) analysis.

\section{Normal moveout analysis}

NMO can be defined as "The additional time required for energy to travel from source to a flat reflecting bed and back to geophone at some distance from the source point compared with the time it takes to return to the geophone at the source point" (Sheriff, 1991). The normal moveout equation used commonly to shift events at non-zero offsets to their equivalent zero offset time and is given by:

$$
t_{x}^{2}=t_{0}^{2}\left(\frac{2 x}{v_{n m o}}\right)^{2}
$$

where $t_{x}$ is the traveltime at offset $\mathrm{x}, t_{0}$ is the zero-offset (normal incidence) traveltime and $v_{n m o}$ is the stacking velocity (Dix, 1955). This is a short offset (2-term) approximation of the Taylor series expansion of traveltime as a function of offset as given by Taner and Koehler (1969) over an isotropic horizontally layered media.

This equation can only estimate short offset velocity; we are forced to throw out the long offset information, which usually contains the anisotropy information. In order to use this long offset information we need to use a longer offset equation; we can use a higher order moveout equation as proposed by Taner, but we will lose the simplicity of hyperbola implementation. To over come this limitation we use SHNMO.

\section{SHIFTED NMO HYPERBOLA EQUATION}

Castle (1994) derives a new approximation to the NMO equation using the principles of reciprocity, finite slowness and exact constant velocity limit. For "reasonable" offsets, his approximation, termed as SHNMO, and can be written in different ways, but the easily comprehensible and implementable form can be written as:

$$
t_{x}=t_{s}+\sqrt{t_{0}+\frac{4 x^{2}}{s . v_{n m o}}}
$$

where $t_{x}$ is the traveltime at an offset $(x), t_{s}$ is the time-shift, $t_{0}$ is the zero-offset time, $v_{n m o}$ is the NMO velocity and $s$ is the shift parameter. In the above equation, the "shift parameter", $s$, is a constant and is described as:

$$
s=\frac{A_{4}^{2}}{A_{2}^{2}},
$$

where $A_{2}$ and $A_{4}$ are the second and fourth order moments of the velocity distribution. Although with a constant $s$ the shifted hyperbola equation fits larger offsets better than Dix 
NMO formula (equation (1)), Castle (1994) showed that by varying the $s$ with offset, one could obtain an exact fit of the traveltime curve. In equation (2) the shift parameter $s$ is a constant. Castle (1994) states that for seismic data at longer offsets we can design $s$ such that it varies with offset. The scale dependent $s$ can be expressed as follows:

$$
s(x)=\frac{1+a x^{2}}{1+b x^{2}}
$$

We have modified this equation into the following form so that it is easy to implement and can be extended to larger offsets in order to account for strong non-hyperbolic moveout. This new offset dependent on offset $s(x)$ can be written as:

$$
s(x)=s+a x^{1}+b x^{2}+c x^{3}+d x^{4} .
$$

By extending the normal-SHNMO equation, which has a constant shift into offset dependent SHNMO, we hope to get a velocity dependent on the offset. The offset dependent SHNMO can be written as:

$$
t_{x}=t_{s(x)}+\sqrt{t_{0}+\frac{4 x^{2}}{s(x) \cdot v_{n m o}}} .
$$

\section{Advantages of SHNMO}

The following are the advantages of SHNMO over the Dix NMO hyperbola:

- Accurate up to fourth order in offset,

- can approximate higher orders in media with "lesser" inhomogeneity,

- easier to implement than other higher order approximations,

- shift parameter provides vital information on the anisotropy and parameters

We will now discuss the procedure for estimation of anisotropy parameters in detail.

\section{ESTIMATION OF $\epsilon$}

$\epsilon$ is the parameter which depends on the difference between both vertical and horizontal vertical phase velocities scaled with vertical velocity. $\epsilon$ can be expressed as:

$$
\epsilon=\frac{v_{v e r t}-v_{h o r}}{2 v_{h o r}}
$$

As a cursory inspection of equation (7) would show that if we have an estimate of both vertical velocity $\left(v_{v e r t}\right)$ and horizontal velocity $\left(v_{\text {hor }}\right)$ we can calculate $\epsilon$. 
Estimation of $v_{v e r t}$ and $v_{h o r}$

The estimation of these velocities has been a persistent problem in successfully estimating the anisotropic parameters both $\epsilon$ and $\delta$. In a single layer model $v_{\text {vert }}$ is the easiest of the two, and can be estimated using the hyperbolic velocity analysis, which being a short offset approximation is good at estimating $v_{v e r t}$; however the problem of estimating $v_{\text {hor }}$ remains. Many researchers proposed various schemes to estimate horizontal velocity; one of the main schemes is to use tomographic inversion at various angles to estimate $v_{\text {hor }}$.

The technique which we propose here fits the traveltime data with a SHNMO curve . As discussed earlier we would be using offset dependent SHNMO (equation(6)) to estimate both far-offset and short-offset velocities. The problem now is to fit this equation with six unknowns $\left(s, a, b, c, d\right.$ and $\left.v_{n m o}\right)$ to traveltime curve; we solve this by using a random-walk technique, simulated annealing (SA).

\section{Implementation}

The SHNMO is a non-linear equation and therefore linear inversion techniques like least square inversion fail. A random walk technique like SA inversion would serve the purpose of inverting the moveout equation for both $s$ and $v_{n m o}$. The offset-traveltime information of each significant reflector is used for this inversion.

The model space to be inverted for here in this case is $m\left(s, v_{n m o}\right)$. One of the advantages of this inversion technique is it gives a good control on the range of solutions in the model space and the acceptable error range.

Simulated Annealing inversion needs an initial guess of the range of model parameters in which the solution falls. First a very broad range of model values is specified as the search window along with a very large acceptable error. This range is refined at each run and also the acceptable error is reduced. This operation is repeated until the error converges at minima acceptable to the user.

\section{Simulated Annealing}

Simulated annealing is a generalization of a Monte Carlo method for examining the equations of state and frozen states of n-body systems (Metropolis et al., 1953) . an annealing process is a process in which a melt, which is initially at high temperature and disordered, is slowly cooled so that the system at any time is approximately in thermodynamic equilibrium.

There are various algorithms used to implement SA. We have implemented the Metropolitan algorithm, proposed by Metropolis et al. (1953). Metropolitan scheme involves the following steps: first an initial state of a thermodynamic system was chosen at energy $E$ and temperature $T$. Holding $T$ constant the initial configuration is perturbed and the change in energy $d E$ is computed. If the change in energy is negative the new configuration is accepted. If the change in energy is positive, it is accepted with a probability given by the Boltzmann factor ( $\exp ^{\frac{-d E}{T}}$ ). This processes is then repeated sufficient times to give good 
sampling statistics for the current temperature, and then the temperature is decremented and the entire process repeated until a frozen state is achieved at $T=0$ (Woollard and Rose, 1963).

\section{Estimation of $v_{v e r t}$ and $v_{h o r}$}

In equation (6) $v_{n m o}$ is not constant and it is determined by $s(x) v_{n m o}$, therefore the NMO velocity is a function of offset. From this function we can determine zero offset velocity which equates to $v_{\text {vert }}$ and far-offset velocity. This far offset velocity can be equated to $v_{\text {hor }}$ if we have long enough offsets.

The estimated $v_{v e r t}$ and $v_{\text {hor }}$ are used to calculate the Thomsen's anisotropy parameter $\epsilon$. As shown earlier $\epsilon$ can be calculated using equation (7).

\section{ESTIMATION OF $\delta$}

The other Thomsen's parameter that needs to be calculated is $\delta$. This parameter $\delta$ controls the depth determination and its determination is essential for depth imaging of anisotropic media.

The relation for $\delta$ in weak anisotropic media can be written as:

$$
\delta=\frac{\left(c_{13}+c_{55}\right)^{2}-\left(c_{33}-c_{55}\right)^{2}}{2 c_{33}\left(c_{33}-c_{55}\right)},
$$

where $c_{i j}$ are the Christoffel's coefficients, which are equal to $a_{i j} \gamma$; where $a_{i j}$ is the velocity if $i j$ direction and $\gamma$ is the density.

$\delta$ can is also be written as the relation between zero-offset velocity $v_{0}$ and the $v_{n m o}$ as :

$$
v_{n m o}=v_{0} \sqrt{1+2 \delta}
$$

The drawback of this relation is that it requires the knowledge of the $v_{0}$. we do not always have the information on the nature of $v_{0}$. Usually the value of $v_{0}$ is obtained from a VSP experiment or a checkshot value which is not usually available. This has been a major obstacle in estimating anisotropy parameters. Alkhalifah and Tsvankin (1995) proposed a parameter $\eta$ to quantify the anisotropy in the media which is easier to estimate and can be used to get better time imaging.

\section{The parameter $\eta$}

The anisotropy parameter $\eta$, a combination of both $\epsilon$ and $\delta$, was introduced by Alkhalifah and Tsvankin (1995) and it can be written as follows:

$$
\eta=\frac{\epsilon-\delta}{1-2 \delta}
$$

By observing the equation (10) it is apparent that that $\eta$ becomes zero when both $\epsilon$ and $\delta$ are equal to each other. In other words $\eta$ can be treated as the measure of anellipticity, referring 
to elliptical Anisotropy . The concept of Elliptical anisotropy was proposed by Thomsen (Thomsen, 1986) where both the parameters of $\epsilon$ and $\delta$ are equal to each other. Therefore in the case of elleptical anisotropy where $\eta=0$ there is no way we can distingguish the elliptical anisotropy from isotropic medium. This is a major limitiaion of this method.

\section{Determination of $\delta$ without the knowledge of $v_{0}$}

As seen in the above section, it is impossible to measure the anisotropy parameter $\delta$ without information $v_{0}$. The following method can be used to overcome this difficulty of prior information of $v_{0}$. The first step in using this method is to estimate the value of $\epsilon$ as described in the above section. This is accomplished by fitting a higher order NMO curve to the travel time data. We fit a SHNMO curve with the shift parameter $s$ varying with offset. The next step involves in relating $s$ to the parameter $\delta$.

\section{Relation between $s$ and $\delta$}

Siliqi and Bousquié (2000) have worked on relating $s$ to the parameter $\eta$. According to them the parameter $\eta$ is related to the $s$ in the following way:

$$
s=1+8 \eta
$$

which was found to be not correct. We found the correct relation to be as follows:

$$
s=1+4 \eta
$$

The derivation of this equation is shown in Appendix 1. Equation (12) can be written as follows:

$$
s=1+4 \frac{\epsilon-\delta}{1-2 \delta}
$$

In Figure 1 equation (13) is plotted with various values of $\epsilon$ and $\delta$. This figure shows how $s$ varies with $\delta$ for $\epsilon$ fixed at -0.2 . Figure 2 shows how $s$ varies with $\delta$ for $\epsilon$ fixed at -0.1 and figure 1 with $\epsilon$ fixed at -0.15 . Using these curves and with a knowledge of $s$ we can determine $\delta$.

\section{ESTIMATION ON A MODEL}

This method is now tested on a single layer VTI model. The data was generated for various values of $\epsilon$ and $\delta$ which are shown in Table 1 .

Table 1. The table comparing Estimated and model anisotropy parameters.

\begin{tabular}{cccccc}
\hline Model & $v_{0}$ & $\epsilon_{\text {model }}$ & $\epsilon_{\text {est }}$ & $\delta_{\text {model }}$ & $\delta_{\text {est }}$ \\
\hline Model 1 & 2000 & 0.2 & 0.176 & -0.1 & 0.077 \\
Model 2 & 2000 & 0.1 & 0.087 & -0.1 & -0.088 \\
Model 3 & 2000 & 0.2 & 0.18 & 0 & 0.052 \\
\hline
\end{tabular}

The data is then sorted into CMP gathers and the traveltime data picked for a CDP gather an the end of the model. Figure 4 shows the moveout curve for Model 1, Figure 1 shows the moveout curve for Model 2 and Figure 6 shows the moveout curve for Model 3. 


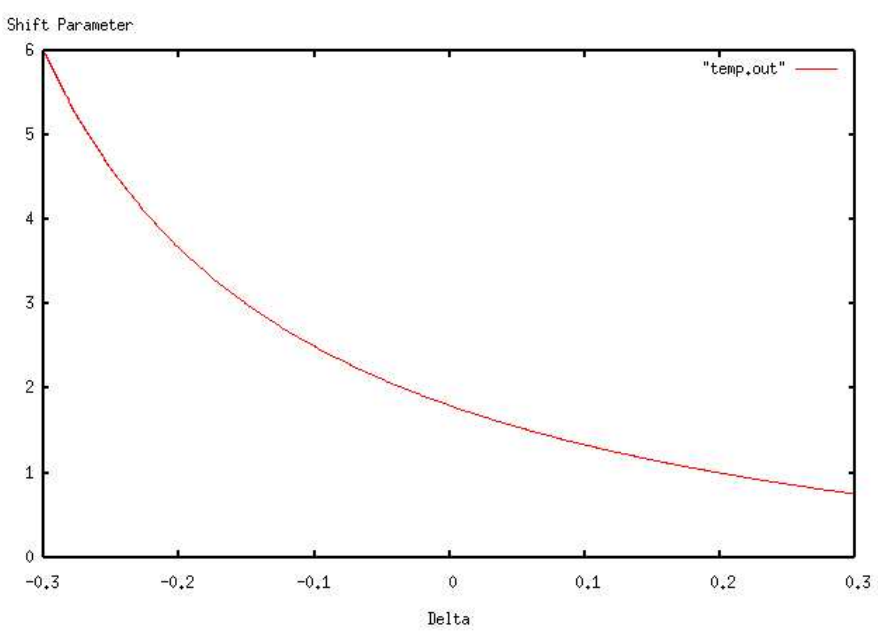

FIG. 1. The plot of $s$ vs. $\delta$ with $\epsilon=-0.2$.

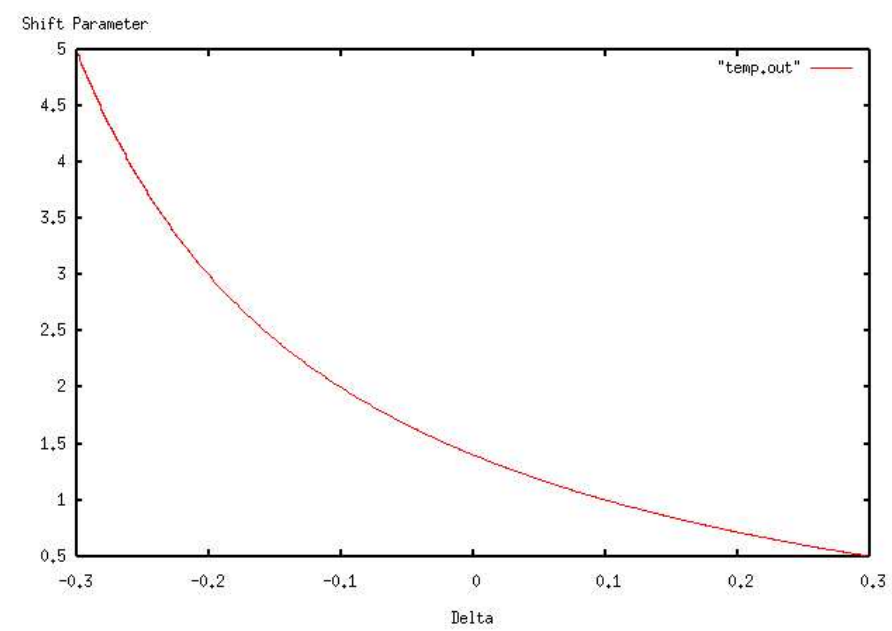

FIG. 2. The plot of $s$ vs. $\delta$ with $\epsilon=-0.1$.

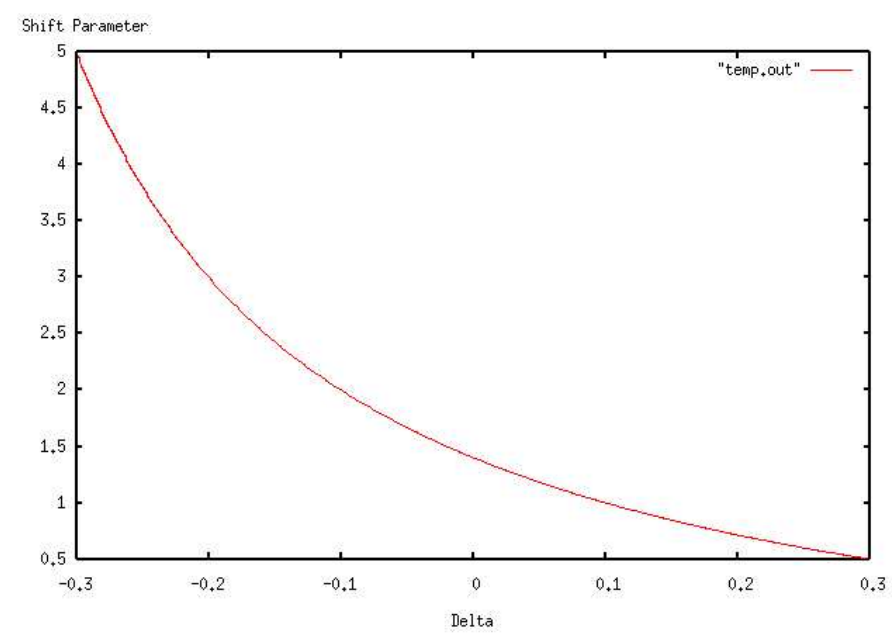

FIG. 3. The plot of $s$ vs. $\delta$ with $\epsilon=-0.15$. 


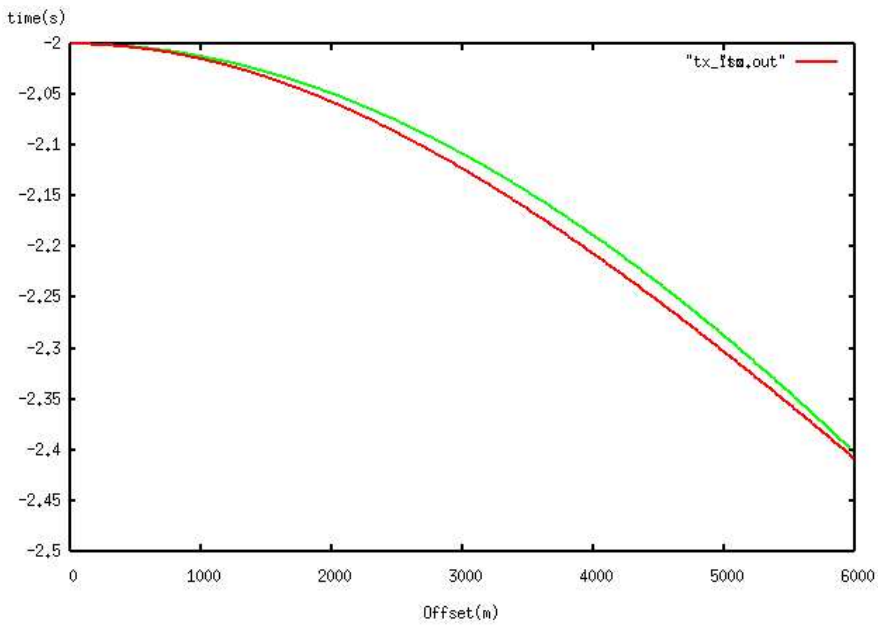

FIG. 4. The moveout for Model 1.

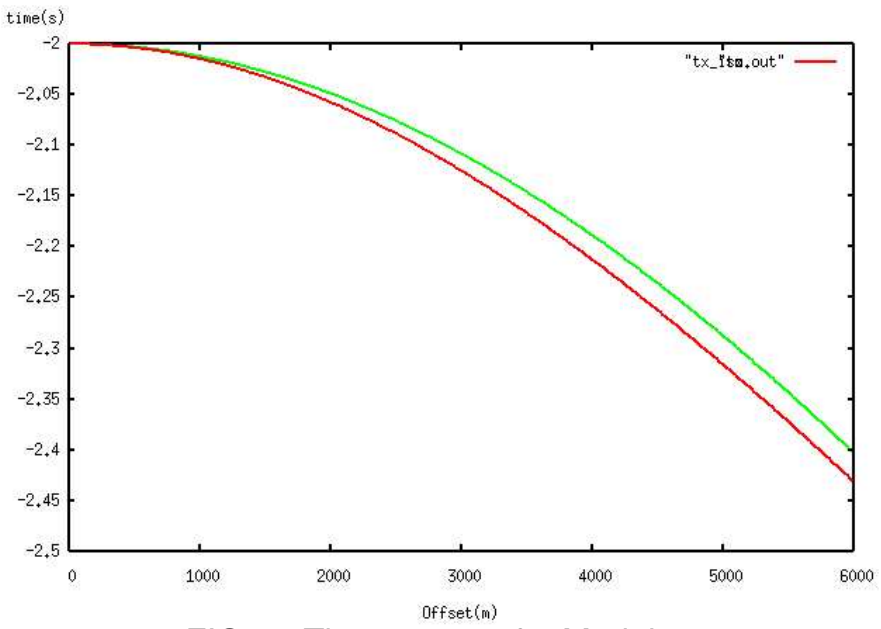

FIG. 5. The moveout for Model 2.

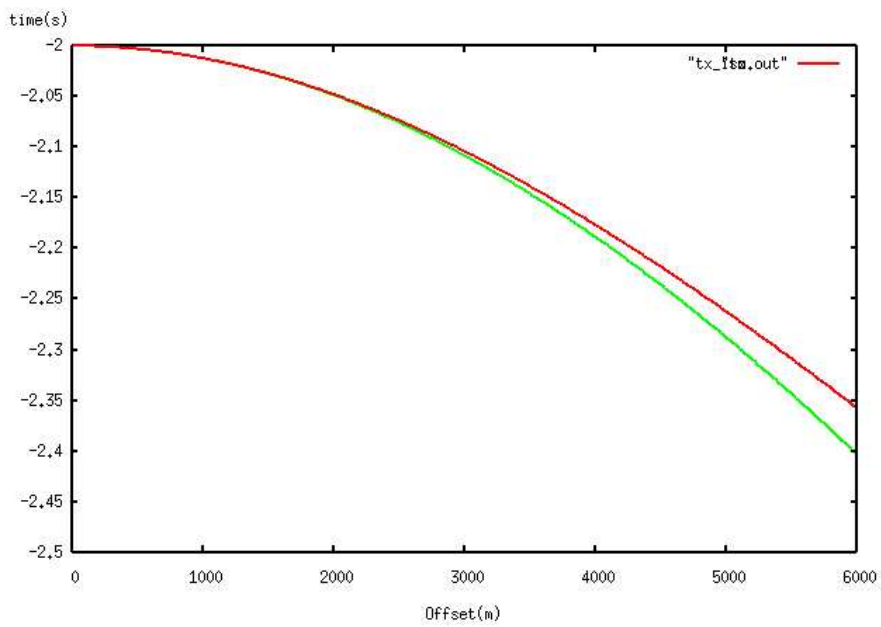

FIG. 6. The moveout for Model 3. 
Simulated annealing is performed on these curves and the parameters are estimated. Figures 7, 8 and 9 show the residual times after the both NMO and SHNMO are applied; the red curve shows the residual time when NMO is applied and the green curve the residual time when SHNMO is applied.

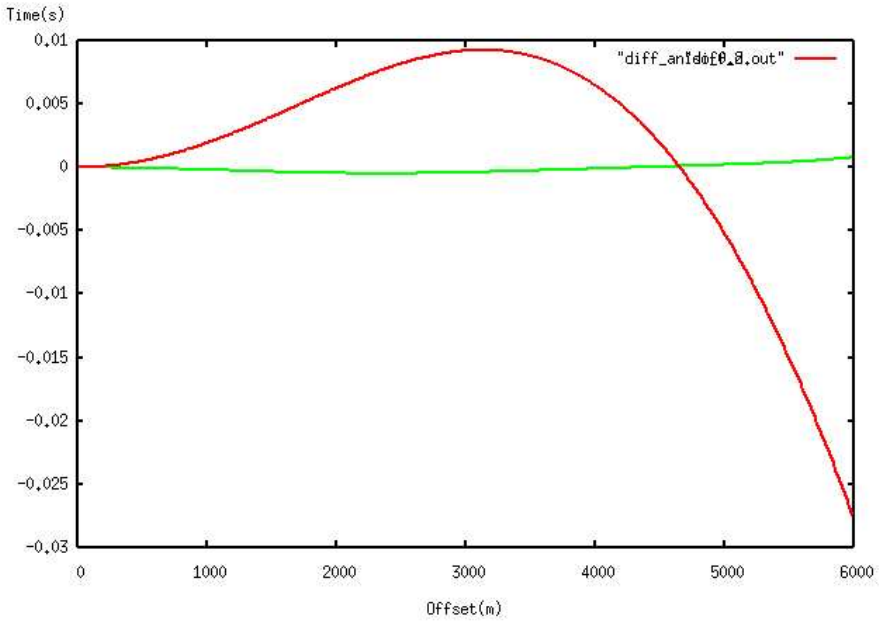

FIG. 7. Snapshot of the wavefront in medium with $\epsilon=0.2$ and $\delta=0.2$.

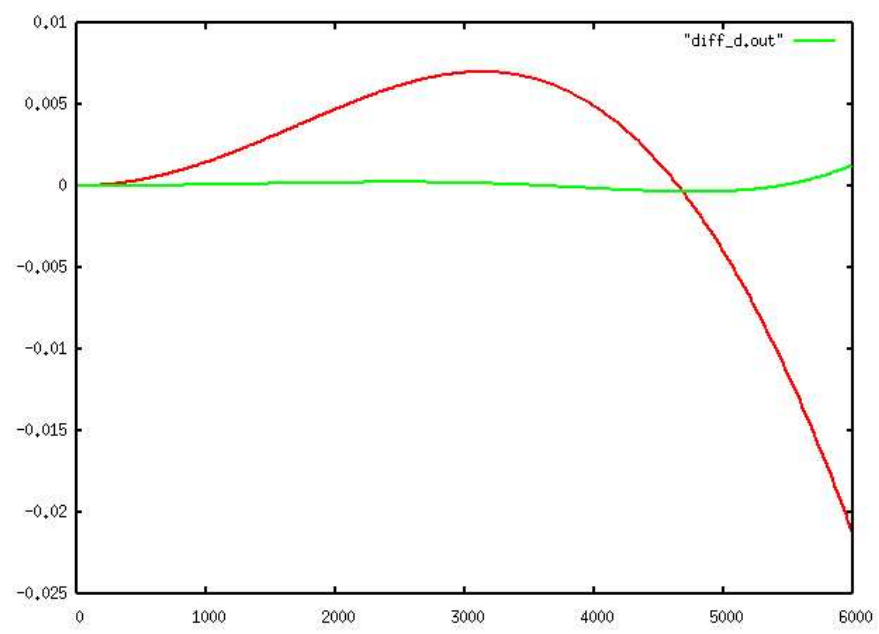

FIG. 8. Snapshot of the wavefront in medium with $\epsilon=0.2$ and $\delta=0.2$.

\section{ESTIMATION OF MODEL PARAMETERS IN ORTHORHOMBIC MEDIUM}

Orthorhombic media, unlike VTI media has three mutually orthogonal planes of mirror symmetry (Tsvankin, 2001). In this section we detail a method which can be used to estimate the anisotropy parameters in orthorhombic media.

\section{Orthorhombic medium}

Nine independent stiffness coefficients are needed to describe this symmetry. The most common example of orthorhombic symmetry in real geology is that of sedimentary basins in a combination of parallel vertical fractures with vertical transverse symmetry in the 


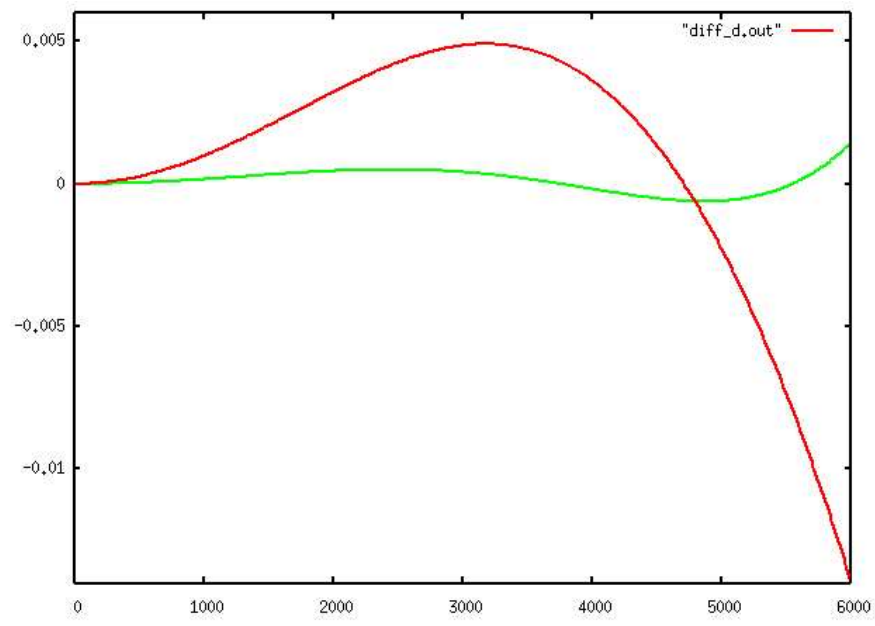

FIG. 9. Snapshot of the wavefront in medium with $\epsilon=0.2$ and $\delta=0.2$.

background. Bakulin et al. (2000) states that orthorhombic symmetry may be the simplest realistic symmetry for many geophysical problems. This symmetry is illustrated in Figure 10 .

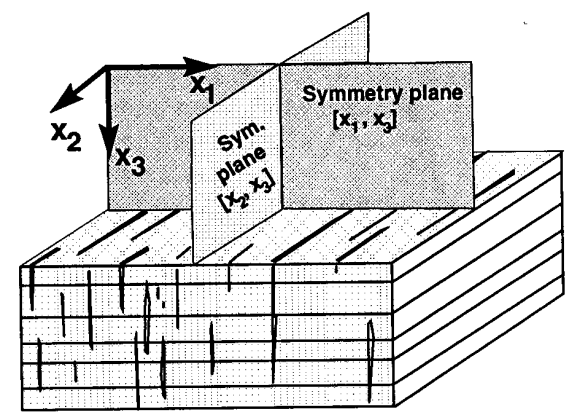

FIG. 10. Orthorhombic symmetry. Courtesy (Tsvankin, 2001)

\section{Stiffness matrix for Orthorhombic medium}

The Christoffel equation for a orthorhombic media can written as follows:

$$
c_{i j}=\left(\begin{array}{cccccc}
c_{11} & c_{12} & c_{13} & 0 & 0 & 0 \\
c_{12} & c_{22} & c_{23} & 0 & 0 & 0 \\
c_{13} & c_{23} & c_{33} & 0 & 0 & 0 \\
0 & 0 & 0 & c_{44} & 0 & 0 \\
0 & 0 & 0 & 0 & c_{55} & 0 \\
0 & 0 & 0 & 0 & 0 & c_{66}
\end{array}\right)
$$

where $c_{i j}$ are the Christoffels coeefcients and are related to the velocities $a_{i j}$ by the following relation:

$$
a_{i j}=c_{i j} \delta
$$

where $\delta$ is the density. 


\section{Anisotropy parameters in Orthorhombic media}

Tsvankin (2001) has introduced anisotropic parameters that characterize a wide range of signatures for orthorhombic anisotropy. They have used the orthorhombic anisotropy's analogy with VTI media to propose these parameters on the lines of Thomsen's parameters for VTI media.

There are five independent parameters which can be used to classify this media, they are: $\epsilon^{(2)}, \delta^{(2)}, \epsilon^{(1)}, \delta^{(1)}$, and $\delta^{(3)}$.

The subscript refers to the normal direction of the symmetry axis; for example the subscript (2) refers to the $x_{2}$ axis, which defines the normal direction to the $\left[x_{1}, x_{3}\right]$ symmetry plane. $\epsilon^{(2)}$ be written as:

$$
\epsilon^{(2)}=\frac{c_{11}-c_{33}}{2 c_{33}}
$$

$\delta^{(2)}$ be written as

$$
\delta^{(2)}=\frac{\left(c_{13}+c_{55}\right)^{2}-\left(c_{33}-c_{55}\right)^{2}}{2 c_{33}\left(c_{33}-c_{55}\right)} .
$$

It can be easily noted that $\epsilon^{(2)}$ and $\delta^{(2)}$ are analogous to the parameters $\epsilon$ and $\delta$ in VTI media. The only difference is the coefficient $c_{44}$ is used to calculate $\delta$ in VTI media, while in Orthorhombic media the coefficient $c_{55}$ is used. The parameters in the $\left[x_{2}, x_{3}\right]$ symmetry plane can be written as:

$$
\epsilon^{(1)}=\frac{c_{22}-c_{33}}{c_{33}}
$$

and $\delta^{(1)}$ can be written as

$$
\delta^{(1)}=\frac{\left(c_{23}+c_{44}\right)^{2}-\left(c_{33}-c_{44}\right)^{2}}{2 c_{33}\left(c_{33}-c_{44}\right)} .
$$

The above four anisotropy parameters can be used to calculate the eight stiffness coefficients: $c_{11}, c_{22}, c_{33}, c_{44}, c_{55}, c_{66}, c_{23}$ and $c_{13}$. The only remaining stiffness $c_{12}$ can be replaced with a dimensionless anisotropic parameter $\delta^{(3)}$ analogous to the coefficients $\delta^{(1)}$ and $\delta^{(2)}$ :

$$
\delta^{(3)}=\frac{\left(c_{12}+c_{66}\right)^{2}-\left(c_{11}-c_{66}\right)^{2}}{2 c_{11}\left(c_{11}-c_{66}\right)}
$$

\section{Estimation of anisotropy parameters}

As we have seen before, the anisotropy parameters $\epsilon^{(2)}$ and $\delta^{(2)}$, which are analogous to VTI parameters, can be estimated using the method discussed above. It is recalled that $\epsilon^{(2)}$ and $\delta^{(2)}$ are the VTI parameters, with the $x_{2}$ axis as the symmetry axis. These parameters can be obtained by an analysis of the data along the $x_{1}$ axis, which is perpendicular to the $x_{2}$ axis, the axis of symmetry.

The parameters $\epsilon^{(1)}$ and $\delta^{(1)}$ are the parameters with $x_{1}$ axis as the symmetry axis. These are the azimuthal anisotropy parameters. They can also be estimated in the same way in the way described above, i.e. by doing the analysis along the $x_{2}$ axis. 
The final parameter $\delta^{(3)}$, which controls the Christoffel's parameter $c_{13}$, is a special parameter. It needs the information of horizontal velocity in the principal direction, which we assumed to be the $x_{1}$ direction. We have discussed the method for estimating the velocity in the principal direction $c_{11}$. The parameter $\delta^{(3)}$ can be estimated using the following equation:

$$
v_{n m o}=v_{11} \sqrt{1+2 \delta^{(3)}} \text {. }
$$

A major challenge in applying this method is deciding the direction of symmetry in the model. The orientation of the model is very important to identify the principal axis of propagation. This being a modelling study the directions of symmetries are known; on the other hand in real data we seldom have this knowledge of the direction of the symmetry axis.

\section{Estimation on a model}

The method is now tested on the same model we used earlier to test the method detailed above in orthorhombic model. The lower layer now has has orthorhombic symmetry. As discussed above it has five parameters $\left(\epsilon^{(2)}, \epsilon^{(2)}, \delta^{(1)}, \delta^{(1)}\right.$ and $\left.\delta^{(3)}\right)$ to characterize it. we generate seismic data over an orthorhombic media, the method detailing the procedure for generating seismic data is detailed in Elapavuluri et al.; the anisotropy analysis is performed on this data.

\section{Model}

The model is $2.5 \mathrm{D}$ model with a single layer; the second layer is orthorhombic. Orthorhombic media have two different directions of symmetry Tsvankin (2001); therefore the estimation of the parameters in orthorhombic media have to estimated in two different different directions orthogonal to each other.

\section{Estimation of $\epsilon^{(2)}$ and $\delta^{(2)}$}

The anisotropy parameters $\epsilon^{(2)}$ and $\delta^{(2)}$ are the anisotropy parameters with the $x_{2}$ axis as the symmetry axis, so in order to estimate these parameters, the traveltime data is analyzed along the $x_{1}$ axis. A CMP point at the end of the model is selected and the traveltime values are picked w.r.t to the offset. Figure shows the traveltime curve w.r.t to the offset. The inversion routine based on simulated annealing is performed on this data, and $s$ and $v_{0}$ and $v_{\text {hor }}$ are estimated. Using these values, $\epsilon^{(2)}$ and $\delta^{(2)}$ are calculated.

\section{Estimation of $\epsilon^{(1)}$ and $\delta^{(1)}$}

The anisotropy parameters $\epsilon^{(1)}$ and $\delta^{(1)}$ are the anisotropy parameters with $x_{1}$ axis as the symmetry axis, so in order to estimate these parameters, the traveltime data is analyzed along the $x_{2}$ axis. The same scheme used in the above section to estimate $\epsilon^{(2)}$ and $\delta^{(2)}$ is used for the estimation of these parameters. The value of $v_{0}$ remains the same, while the $v_{\text {hor }}$ becomes $c_{22}$ rather than $c_{11}$. 


\section{Estimation technique}

As discussed earlier, the traveltime is picked in the $x_{1}$ direction and also $x_{2}$ direction. The Figures 11 and 12 show the moveout curves for Model1 in the these directions respectively; green curve the moveout if the medium were isotropic and the red curve for the anisotropic case. The Table 2 shows the values of $s, v_{0}, v_{h o r}, \epsilon^{(2)}$ and $\delta^{(2)}$ in $x_{1}$ direction.

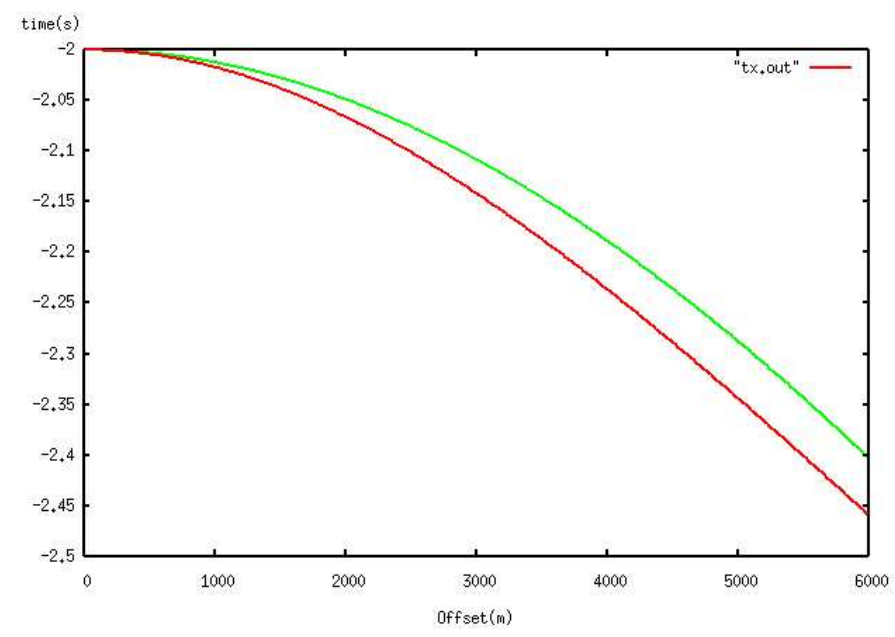

FIG. 11. Traveltime moveout in the $x_{2}$ axis.

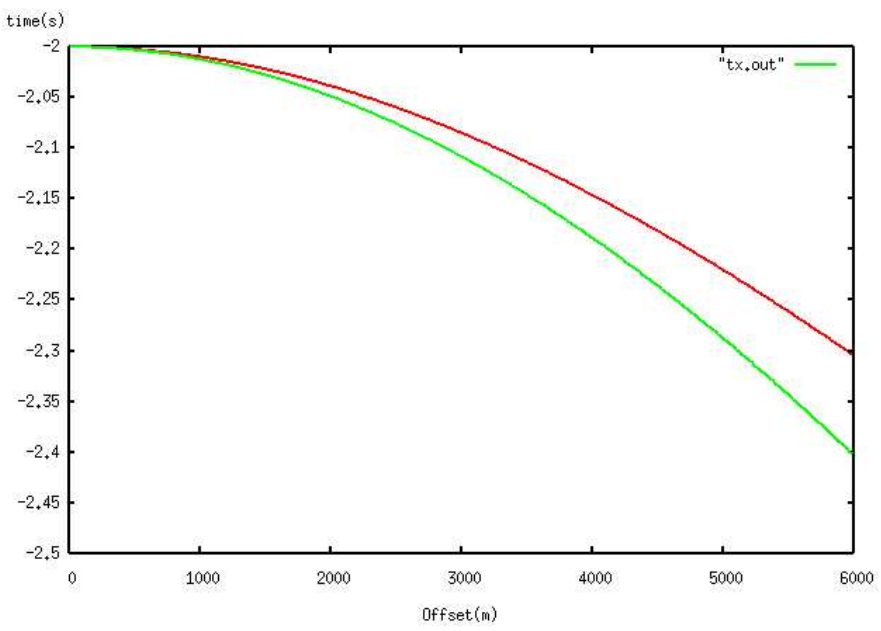

FIG. 12. Traveltime moveout in the $x_{1}$ axis.

The Table 3 shows the values of $s, v_{0}, v_{h o r}, \epsilon^{(1)}$ and $\delta^{(1)}$ in $x$ direction. These four parameters $\left(\epsilon^{(1)}, \delta^{(1)}, \epsilon^{(1)}\right.$ and $\left.\delta^{(1)}\right)$ are used to estimate the stiffness coefficients, $\left(c_{11}, c_{22}, c_{33}, c_{13}\right.$ and $c_{23}$ ). The only coefficient left to be calculated is $c_{12}$. As discussed,this stiffness coefficient can be calculated using the parameter $\delta^{(3)}$, using equation (21).

Table 2. Comparison of estimated and model anisotropy parameters along the $x_{2}$ axis.

\begin{tabular}{cccccc}
\hline Model & $v_{0}$ & $\epsilon_{\text {model }}^{(2)}$ & $\epsilon_{\text {est }}^{(2)}$ & $\delta_{\text {model }}^{(2)}$ & $\delta_{\text {est }}^{(2)}$ \\
\hline Model 1 & 2000 & 0.2 & 0.1777 & -0.2 & -0.1888 \\
Model 2 & 2000 & 0.1 & 0.087 & -0.1 & -0.088 \\
\hline
\end{tabular}




\begin{tabular}{cccccc}
\hline Model & $v_{0}$ & $\epsilon_{\text {model }}^{(2)}$ & $\epsilon_{\text {est }}^{(2)}$ & $\delta_{\text {model }}^{(2)}$ & $\delta_{\text {est }}^{(2)}$ \\
\hline Model 3 & 2000 & 0.1 & 0.1777 & 0.0 & 0.017 \\
\hline
\end{tabular}

Table 3. Comparison of estimated and model anisotropy parameters along the $x_{1}$ axis.

\begin{tabular}{cccccc}
\hline Model & $v_{0}$ & $\epsilon_{\text {model }}^{(2)}$ & $\epsilon_{\text {est }}^{(2)}$ & $\delta_{\text {model }}^{(2)}$ & $\delta_{\text {est }}^{(2)}$ \\
\hline Model 1 & 2000 & 0.1 & 0.0768 & 0.0 & 0.017 \\
Model 2 & 2000 & 0.2 & 0.189 & -0.2 & -0.189 \\
Model 3 & 2000 & 0.15 & 0.1777 & -0.1 & -0.078 \\
\hline
\end{tabular}

\section{CONCLUSIONS}

In this paper we have presented a scheme to estimate anisotropy parameters in generalized anisotropic media. We have applied this method on both VTI, which needs only 2 parameters to be characterized, and also to orthorhombic media which on the other hand needs 5 parameters. We have shown that the limitation of requiring knowledge of $v_{0}$ for the estimation of the anisotropy parameters can be overcome using the additional information obtained by using a shifted hyperbola with offset dependent shift parameter. The major limitation of this method is, it needs the moveout to be hyperbolic. In case of elliptical anisotropy, where the medium is anisotropic yet the moveout os hyperbolic, this method fails to estimate the anisotropy parametes.

This method is then tested on data generated over both VTI and orthorhombic models. This method will be tested on real data to test the effectiveness of this technique.

\section{REFERENCES}

Alkhalifah, T., and Tsvankin, I., 1995, Velocity analysis for transversely isotropic media: Geophysics, 60, No. 05, 1550-1566.

Alkhalifah, T. A., and Tsvankin, I., 1994, Velocity analysis for transversely isotropic media, in 64th Ann. Internat. Mtg, Soc. of Expl. Geophys., 1000-1003.

Bakulin, A., Grechka, V., and Tsvankin, I., 2000, Estimation of fracture parameters from reflection seismic data - Part I: HTI model due to a single fracture set: Geophysics, 65, No. 6, 1788-1802.

Castle, R. J., 1994, Theory of normal moveout: Geophysics, 59, No. 06, 983-999.

Dix, C. H., 1955, Seismic velocities from surface measurements: Geophysics, 20, No. 01, 68-86.

Metropolis, N., Rosenbluth, M., Teller, A., and Teller, E., 1953, Equation of state calculations by fast computing machines: J. Chem Phys, 21, 1087-1092.

Sheriff, R. E., 1991, Encyclopedic Dictionary of Exploration Geophysics: Soc. of Expl. Geophys., how it was done? - see TLE-10-4-41-42.

Siliqi, R., and Bousquié, N., 2000, Anelliptic time processing based on a shifted hyperbola approach, in 70th Ann. Internat. Mtg, Soc. of Expl. Geophys., 2245-2248.

Taner, M. T., and Koehler, F., 1969, Velocity spectra - Digital computer derivation and applications of velocity functions: Geophysics, 34, No. 06, 859-881.

Thomsen, L., 1986, Weak elastic anisotropy: Geophysics, 51, No. 10, 1954-1966. 
Tsvankin, I., 2001, Seismic Signatures and Analysis of Reflection Data in Anisotropic Media: Pergamon, how it was done? - see TLE-10-4-41-42.

Woollard, G. P., and Rose, J. C., 1963, International Gravity Measurements: Soc. of Expl. Geophys.

\section{APPENDIX A}

In this appendix we will derive an equation to relate $s$ and $\eta$. According to Castle (1994) $s$ can be written as:

$$
s=\frac{A_{2}^{4}}{A_{4}^{4}},
$$

where $A_{2}$ is the 2-order moveout and $A_{4}$ is the fourth order moveout. On the other hand, according to Alkhalifah and Tsvankin (1995) $\eta$ can be written as

$$
v_{n m o}=v_{h o r} \sqrt{1+2 \eta}
$$

We know that $v_{n m o}$ is the second order moveout and is therefore equal to $A_{2}$ and in the similar fashion $v_{h o r}$ is approximately equal to the fourth order moveout, $A_{4}$. Applying these relations and equating equations A-1 and A-2 we get the following relation:

$$
s=(1+2 \eta)^{2} .
$$

Expanding equation (A-3) and neglecting the higher order terms we get the final relation as:

$$
s=1+4 \eta
$$

